Rezension : Kurt W. Schmidt, Markus Sold, Torsten Verrel (Hrsg) (2012) Zum Umgang mit Behandlungsfehlern. (Organisations-)Ethische, rechtliche und psychosoziale Aspekte. Tagungsband der ELSA-Klausurwoche 2012

\author{
Monteverde, Settimio
}

DOI: https://doi.org/10.1007/s00481-013-0276-2

Posted at the Zurich Open Repository and Archive, University of Zurich ZORA URL: https://doi.org/10.5167/uzh-84509

Journal Article

Published Version

Originally published at:

Monteverde, Settimio (2014). Rezension : Kurt W. Schmidt, Markus Sold, Torsten Verrel (Hrsg) (2012) Zum Umgang mit Behandlungsfehlern. (Organisations-)Ethische, rechtliche und psychosoziale Aspekte. Tagungsband der ELSA-Klausurwoche 2012. Ethik in der Medizin, 26(1):83-84.

DOI: https://doi.org/10.1007/s00481-013-0276-2 


\title{
Kurt W. Schmidt, Markus Sold, Torsten Verrel (Hrsg) (2012) Zum Umgang mit Behandlungsfehlern. (Organisations-)Ethische, rechtliche und psychosoziale Aspekte. Tagungsband der ELSA-Klausurwoche 2012
}

\author{
Reihe Ethik \& Recht im Krankenhaus, Bd. 1, Lit-Verlag, \\ Berlin, 318 Seiten, 29,90 €, ISBN 978-3-643-11910-0
}

\section{Settimio Monteverde}

Online publiziert: 15. September 2013

(c) Springer-Verlag Berlin Heidelberg 2013

Die allgemeine Einsicht, dass Irren menschlich ist, sieht sich in den Handlungsfeldern der Medizin mit dem eigentümlichen Paradox konfrontiert, dass hier Fehler in der Regel zum Bereich des Unplanbaren und Unerwarteten gehören, die dem Anspruch der Medizin als exakter Handlungswissenschaft nicht genügen. Aber trotzdem geschehen sie täglich. Nur vor dem Hintergrund dieses Anspruchs und dieser Erwartung an die Medizin wird verständlich, wieso das Dokument des US Institute of Medicine „To Err is Human“ im Jahre 2000, das erstmals umfangreiche Zahlen von Todesfällen aufgrund von Behandlungsfehlern lieferte, auf ein derart großes Echo stieß und im Gefolge zahlreiche Patient Safety-Initiativen ins Leben rief. In Einrichtungen des Gesundheitswesens werden seither vermehrt Fehlermeldesysteme eingeführt und ausgebaut. Sie haben das Ziel, über die Erfassung der Umstände der Fehlerentstehung zu systemisch orientierten Ansätzen der Fehlervermeidung zu führen. Als Beispiel dient dazu das in der operativen Medizin vielerorts eingeführte Critical Incident Reporting System (CIRS), das auf Erkenntnissen aus dem nichtmedizinischen Sektor wie z. B. der Aviatik aufbaut. Doch verglichen mit der Fokussierung auf die zunehmend erforschten Mechanismen der Fehlerentstehung ist ein angemessener Umgang mit erfolgten Fehlern - über allfällige strafrechtliche Folgen hinaus - vielerorts ein Desiderat.

Der von Kurt W. Schmidt, Markus Sold und Torsten Verrel herausgegebene Sammelband vereinigt Beiträge, die die Brücke schlagen zwischen diesen beiden Pfeilern einer nachhaltigen Fehlerkultur. Die Beiträge entstammen der gleichnamigen ELSA-Klausurwoche, die 2012 in Bad Homburg stattgefunden hat und in deren Rahmen Forschende aus verschiedenen Bereichen wie Medizin, Recht, Theologie, Philosophie, Pflegewissenschaft, Psychologie und Soziologie empirische, konzeptuelle und normative Aspekte der Thematik diskutierten. Den einzelnen Beiträgen stellen die Herausgeber zehn Thesen voran (S. 17-20), die zentrale Grundannahmen der nachfolgenden Beiträge konkretisieren, systematisch einordnen und

S. Monteverde, MME, MAE, lic.theol, RN ( $\square)$

Zürich, Schweiz

E-Mail: settimio.monteverde@uzh.ch 
beim Lesen die Orientierung erleichtern, gerade auch angesichts zahlreicher Redundanzen in Fragen der Definition und Systematisierung von Behandlungsfehlern.

Die 15 Beiträge sind in vier Abschnitte gegliedert: Grundprobleme einer Fehlerkultur Kommunikation von Behandlungsfehlern - Ethische, theologische und philosophische Aspekte - Strafrechtliche und haftungsrechtliche Aspekte. Diese Strukturierung ist jedoch angesichts der interdisziplinären Ausrichtung zahlreicher Beiträge nicht starr, sondern bezieht sich vorwiegend auf den disziplinären Hintergrund der Autorinnen und Autoren. Sie steckt den Horizont ab, in dem sich die heutige Forschung international bewegt. Begriffliche Differenzierungen aus der sogenannten Human Factors-Forschung nehmen einen wichtigen Platz in mehreren Beiträgen ein. Resultate einer exemplarischen Datenerhebung über strafrechtliche Ermittlungen bei vermuteten Behandlungsfehlern werden genauso diskutiert wie konzeptionelle Fragen am Beispiel der Palliativmedizin. Darüber hinaus werden kommunikative Aspekte thematisiert, die ein kritisches Licht auf das ,traditionelle Fehlermodell“ (S. 102) werfen. Dieses Modell läuft Gefahr, dem „first victim“ (normalerweise der Patient, der einen Behandlungsfehler erleidet) durch Systemversagen ein ,second victim“ in der Gestalt der Arzt- oder einer anderen Fachperson hinzuzufügen (S. 113). Eine „Fehlerkultur" wird skizziert, die kognitions- und verhaltenspsychologische Aspekte integriert. In einem anderen Beitrag werden die kulturelle Bedingtheit von Wahrnehmungen von Patienten und Ärzten bezüglich des Umgangs mit Fehlern empirisch belegt. Auch pflegeethische, theologische und moralphilosophische Dimensionen werden erörtert, die um die Begriffe Schuld, Vergebung und Verzeihung kreisen. Unter Berücksichtigung der Verteilungsproblematik führt der Beitrag von Michael Rosentreter an die Grenzen dessen, was der Begriff des Behandlungsfehlers begrifflich leisten kann: „Quantitativ nicht fassbar sind die unzähligen Lässigkeiten, Unterlassungen und Bedenkenlosigkeiten, die in komplexen Systemen unter hoher Arbeitsdichte unvermeidbar zu sein scheinen und die in ihrer Summe ebenso nachhaltig schädigen wie ,der eine Fehler“" (S. 124). Damit ist eine normative Spannung umschrieben, die nicht in allen Beiträgen des Bandes in gleichem Maße erkennbar ist, aber für das Thema konstitutiv ist.

Gerade wegen seines konsequent interdisziplinären Ansatzes, der vorangestellten Thesen und der wissenschaftlichen Orientierung der Beiträge kann das Buch für diejenigen empfohlen werden, welche in der Einführung von Modellen der Fehlerprävention und des Fehlermanagements tätig sind oder in der diesbezüglichen Begleitforschung. 